\title{
Padronização do tempo de homogeneização na produção de cápsulas magistrais
}

Standardization of homogenization time in the production of capsules in magistral pharmacy

\author{
I. G. Matos²; Y. L. Lima'; Y. M. B. G. Carvalho²; B. S. Lima²; A. A. S. Araújoº; C. \\ M. Lima ${ }^{1}$; M. R. Serafini ${ }^{2 *}$ \\ ${ }^{1}$ Departamento de Farmácia, Universidade Federal de Sergipe, CEP: 49400-000, Lagarto, Sergipe, Brasil.
}

${ }^{2}$ Departamento de Farmácial Laboratório de Ensaios Farmacêuticos e Toxicidade, Universidade Federal de Sergipe, CEP: 49100-000, São Cristóvão, Sergipe, Brasil.

*maiserafini@hotmail.com

(Recebido em 19 de julho de 2018; aceito em 26 de fevereiro de 2019)

\begin{abstract}
A segurança e eficácia de medicamentos produzidos em farmácias magistrais estão relacionadas diretamente com a uniformidade das doses unitárias. O gral e o saco plástico são métodos bastante utilizados para garantir a homogeneização de pós na produção de cápsulas, contudo, não há estudos relacionados a padronização do tempo de homogeneização. Diante disso, o presente estudo teve como objetivo padronizar o tempo de homogeneização na produção de cápsulas de paracetamol, fármaco este atualmente prescrito com grande frequência. $\mathrm{O}$ paracetamol e seus excipientes foram pesados e submetidos a homogeneização em gral (HG) e em saco plástico (HSP), nos tempos de 1, 5 e 10 minutos. Posteriormente, de acordo com a Farmacopeia Brasileira $5^{a}$ edição, o teor de paracetamol presente em cada amostra foi quantificado por cromatografia líquida de alta eficiência (CLAE), em seguida as cápsulas de paracetamol foram manipuladas e submetidas aos testes de peso médio e dissolução. Os resultados mostraram que para HG e HSP nos diferentes tempos, o teor de paracetamol foi satisfatório, sendo superior ao mínimo preconizado de $98 \%$. As cápsulas apresentaram variação individual de peso permitida de $\pm 7,5 \%$ em relação ao peso médio, sugerindo uniformidade das doses. No teste de dissolução, mais de $80 \%$ do paracetamol foi liberado no tempo de 30 minutos. Logo, todos os métodos e tempos de homogeneização poderiam ser utilizados como padrão pela farmácia magistral, pois apresentaram teor de paracetamol dentro do limite preconizado.

Palavras-chave: paracetamol, gral, saco plástico, tempo de homogeneização.
\end{abstract}

The safety and efficacy of medicines produced in pharmacies are directly related to the uniformity of dosage units. The mortar and the plastic bag are widely used methods to ensure homogenization after the production of capsules, however, there are no studies regarding the standardization of homogenization time. Therefore, this study aimed to standardize the homogenization time in the production of paracetamol capsules drug that is currently prescribed for the treatment of many diseases. Paracetamol and its excipients were weighed and subjected to homogenization in a mortar (HM) and plastic bags (HPB) in times of 1, 5 and 10 minutes. Subsequently, according to Brazilian Pharmacopoeia's 5th Edition, the present paracetamol content in each sample was quantitated by high-performance liquid chromatography (HPLC), then paracetamol capsules were handled and subjected to medium weight and dissolution tests. The results showed that for HM and HPB in different times, paracetamol content was satisfactory and above the recommended minimum of $98 \%$, the capsules presented individual weight variation permitted of $\pm 7.5 \%$ over the average weight, suggesting uniformity of doses. In the dissolution test, more than $80 \%$ of the paracetamol was released in 30 minutes. Therefore, all methods and times of homogenization are suitable to be used as standard by the master pharmacy, as they presented paracetamol content within the recommended range.

Keywords: paracetamol, mortar, plastic bag.

\section{INTRODUÇÃO}

A manipulação de medicamentos em farmácias magistrais constitui uma parte integral da prática farmacêutica [1]. A procura por medicamentos manipulados tornou-se uma alternativa ao medicamento de referência, devido à redução de custos, a possibilidade de individualização e personalização da dose e a grande variedade de formas farmacêuticas [2]. A RDC n 67/2007 define manipulação como conjunto de operações farmacotécnicas, com a finalidade de elaborar 
preparações magistrais e oficinais e fracionar especialidades farmacêuticas para uso humano [3]. Em 2008 a RDC 67 foi complementada pela RDC 87, que alterou a submissão de análises de uniformidade de conteúdo e teor de ativos para ao menos uma vez a cada dois meses para cápsulas com menos de $25 \mathrm{mg}$ de ativo e exigiu a padronização de excipientes [4]. Diante disto, a Agência Nacional de Vigilância Sanitária (ANVISA) tem exigido maior rigor nos procedimentos de manipulação de medicamentos, objetivando a padronização e consequentemente a segurança e eficácia destes $[3,4,5,6,7]$.

Dentre as formas farmacêuticas sólidas de uso oral produzidas nas farmácias magistrais, destacam-se as cápsulas duras, que por sua facilidade de formulação e versatilidade, permitem a manipulação em doses individualizadas [8]. Para manipulação de medicamentos na forma farmacêutica cápsula é necessário que se leve em consideração as características físicas, químicas e biológicas de todos os fármacos e excipientes utilizados, como também, o processo de mistura, que deve ocorrer de forma homogênea, sem separação das partículas, misturando pós com características semelhantes de tenuidade e densidade [9].

Dentre os principais fatores que afetam a uniformidade de uma mistura de pós, podemos destacar: a distribuição do tamanho das partículas, os adjuvantes empregados e a proporção entre o tempo e a velocidade da mistura [10]. O método gral (HG) é um meio de mistura de pós que consiste na homogeneização em gral com pistilo com movimentos do centro para a periferia e da periferia para o centro. Este método de homogeneização é um dos mais tradicionais nas farmácias magistrais e é bem descrito na literatura [9]. No entanto, métodos alternativos vêm surgindo nas farmácias magistrais com o intuito de trazer agilidade e praticidade. Destaca-se o método de saco plástico (HSP), que consiste na homogeneização dos pós em sacos plásticos por meio da movimentação manual [11].

Para avaliar a eficiência de homogeneização de uma mistura de pós para manipulação de medicamentos na forma farmacêutica cápsula, são empregados testes farmacopeicos para determinar o teor do princípio ativo e a uniformidade de doses. Porém, nas farmácias magistrais torna-se inviável a realização destes testes em todas as formulações manipuladas, sendo importante para garantir a uniformidade de doses, procedimentos padronizados e eficientes [12].

Sendo assim, torna-se necessário avaliar a eficiência dos métodos de mistura utilizados pelas farmácias magistrais, tais como: HG e HSP e padronizar o tempo de homogeneização. Diante disso, o presente estudo teve como objetivo, padronizar o tempo de homogeneização de cápsulas de paracetamol $(250 \mathrm{mg})$ manipuladas em farmácia magistral, tendo em vista a importância desse parâmetro na qualidade do medicamento manipulado, como também, por esse fármaco ser prescrito com grande frequência.

\section{MATERIAL E MÉTODOS}

\subsection{MATERIAL}

Paracetamol (Purifarma, lote 1451064), MICROCEL ${ }^{\circledR}$ (SM Empreendimentos, lote 14116085); AEROSIL ${ }^{\circledR}$ (Henrifarma, lote 154031814); talco (Henrifarma, lote N-150903); laurilsulfato de sódio (All Chemistry do Brasil, lote ALL 057002); amido de milho (Henrifarma, lote 19953).

\subsection{MÉTODOS}

A uniformidade de uma mistura de pós influencia diretamente na biodisponibilidade e consequentemente eficácia de uma forma farmacêutica. A fim de garantir a uniformidade da mistura de pós alguns fatores como a distribuição do tamanho das partículas, a velocidade de mistura e a proporção entre fármaco e excipientes devem ser levados em consideração.

A HG é um método de mistura para pós que consiste na homogeneização em gral com pistilo com movimentos do centro para a periferia e da periferia para o centro. Este método de homogeneização é um dos mais tradicionais nas farmácias magistrais e é bem descrito na literatura. 
No entanto, métodos alternativos vêm surgindo nas farmácias magistrais com o intuito de trazer agilidade e praticidade. Destaca-se o método HSP, que consiste na homogeneização dos pós em sacos plásticos por meio da movimentação manual.

\subsubsection{MANIPULAÇÃO DAS CÁPSULAS}

As cápsulas foram preparadas pelo método de nivelamento, em encapsuladora manual, após a pesagem e homogeneização dos constituintes (paracetamol e excipientes). A dose de paracetamol estipulada para cada cápsula foi de $250 \mathrm{mg}$. Pesou-se $5 \mathrm{~g}$ do fármaco e $3 \mathrm{~g}$ dos excipientes (MICROCEL ${ }^{\circledR} 5 \%$, AEROSIL ${ }^{\circledR} 1 \%$, talco $30 \%$, lauril sulfato de sódio $2 \%$ e amido qsp) para produzir 20 cápsulas. As matérias-primas (fármaco e excipientes) foram pesadas em balança Gehaka ${ }^{\circledR}$ modelo AG200 e submetidas ao processo de homogeneização, seguindo o princípio da diluição geométrica ou lei da proporcionalidade, conforme descrito na Farmacopeia $5^{\mathrm{a}}$ edição.

Foram preparadas seis placas com vinte cápsulas cada, para avaliar os métodos (HG e HSP) e os diferentes tempos de homogeneização, 1, 5 e 10 minutos. Para a HG foi realizado a pesagem do paracetamol e dos excipientes, que foram transferidos para um gral de porcelana. A homogeneização ocorreu nos tempos de 1, 5 e 10 minutos com auxílio do pistilo, com movimentos do centro para a periferia e da periferia para o centro, em seguida cada amostra foi encapsulada. $\mathrm{O}$ mesmo procedimento foi realizado para a HSP, sendo que após pesagem, os pós foram transferidos para um saco plástico e submetidos a movimentos leves em todas as direções.

\subsubsection{PESO MÉDIO DAS CÁPSULAS DE PARACETAMOL}

Para avaliação do peso médio, 20 cápsulas de paracetamol de cada método e tempo de homogeneização foram pesadas individualmente em balança semi-analítica (GEHAKA ${ }^{\circledR}$ modelo AG200) ligada ao processador estatístico GEHAKA ${ }^{\circledR}$. O programa calcula o peso médio, limites de variação, desvio padrão e o coeficiente de variação, parâmetros estes descritos na Farmacopeia Brasileira $5^{\text {a }}$ edição. Após a realização do controle de qualidade, as cápsulas de paracetamol foram acondicionadas em potes de plástico branco contendo sílica e algodão e devidamente rotulados.

\subsubsection{PERFIL DE DISSOLUÇÃO DAS CÁPSULAS DE PARACETAMOL}

O teste de dissolução das cápsulas de paracetamol foi realizado em triplicata e conforme descrito na Farmacopeia Brasileira $5^{\text {a }}$ edição volume 2 [13]. Foi utilizado o equipamento dissolutor da Nova Ética ${ }^{\circledR}$ modelo 299/6 equipado com dispositivo de cestas. As cápsulas de paracetamol de cada método e tempo de homogeneização, foram submetidas a agitação de $50 \mathrm{rpm}$ durante 30 minutos a $37{ }^{\circ} \mathrm{C}$. Alíquotas de $10 \mathrm{ml}$ de cada amostra foram retiradas, filtradas e diluídas em água/metanol (75:25) até concentração de $10 \mu \mathrm{g} / \mathrm{ml}$. Em seguida, foi quantificado por CLAE. O teor de paracetamol presente nas soluções resultantes, que de acordo com a Farmacopeia Brasileira $5^{\text {a }}$ edição, não poderia ser menor que $80 \%$ (Q) da quantidade declarada de paracetamol dissolvido durante o tempo de 30 minutos.

\subsubsection{DOSEAMENTO DO PARACETAMOL}

Para obtenção da solução padrão e das soluções amostras, $10 \mathrm{mg}$ de paracetamol, HG e HSP nos tempos de 1, 5 e 10 minutos foi pesado e transferido separadamente para balão volumétrico de $100 \mathrm{ml}$ contendo água e metanol (75:25), em seguida as soluções foram submetidas ao banho ultrassônico durante 10 minutos e a solução padrão foi diluída até as concentrações de 2, 4, 6, 8 e $10 \mu \mathrm{g} / \mathrm{ml}$ para obtenção da curva de calibração. O teor de paracetamol nas amostras foi quantificado por cromatografia líquida de alta eficiência (CLAE) de acordo com a Farmacopeia Brasileira $5^{\text {a }}$ edição [13]. 


\section{RESULTADOS E DISCUSSÃO}

\subsection{PESO MÉDIO DAS CÁPSULAS DE PARACETAMOL}

Os resultados obtidos na análise de peso médio das cápsulas de paracetamol (Tabela 1) apresentaram-se satisfatórios em todos os métodos e tempos de homogeneização, tendo em vista que a variação individual de peso permitida para cápsulas duras com peso superior a $300 \mathrm{mg}$ é de $\pm 7,5 \%$, em relação ao peso médio, podendo duas cápsulas excederem estes limites e, nenhuma ultrapassar o dobro dos limites [13].

Tabela 1: Peso médio das cápsulas de paracetamol.

\begin{tabular}{ccccccc}
\hline Parâmetros & \multicolumn{3}{c}{ HG } & \multicolumn{3}{c}{ HSP } \\
\hline Tempos (minutos) & $1{ }^{\prime}$ & 5, & $10^{\prime}$ & $1{ }^{\prime}$ & $5^{\prime}$ & $10^{\prime}$ \\
Peso médio (g) & 0,49 & 0,48 & 0,48 & 0,48 & 0,48 & 0,48 \\
Coeficiente de variação & 1,66 & 2,08 & 1,83 & 2,02 & 1,96 & 2,47 \\
$(\%)$ & 7,50 & 7,50 & 7,50 & 7,50 & 7,50 & 7,50 \\
Variação permitida (\%) & 0,50 & 0,50 & 0,49 & 0,49 & 0,50 & 0,50 \\
Peso máximo & 0,47 & 0,47 & 0,46 & 0,46 & 0,46 & 0,46 \\
Peso mínimo & 0,52 & 0,52 & 0,51 & 0,52 & 0,52 & 0,52 \\
Peso máximo permitido & 0,45 & 0,45 & 0,44 & 0,44 & 0,45 & 0,44 \\
Peso mínimo permitido & 0,4 & & & & &
\end{tabular}

\subsection{PERFIL DE DISSOLUÇÃO DAS CÁPSULAS DE PARACETAMOL}

Todos os métodos e tempos de homogeneização resultaram em perfis de dissolução satisfatórios (Tabela 2), com liberação do paracetamol superior a $80 \%$ no tempo de 30 minutos, valor mínimo preconizado pela Farmacopeia Brasileira $5^{a}$ edição [13]. Sendo assim, os métodos e tempos de homogeneização demonstram-se viáveis para utilização pela farmácia magistral, pois apresentaram liberação do paracetamol superior a $80 \%$ no tempo de 30 minutos.

Tabela 2: Perfil de dissolução das cápsulas de paracetamol

\begin{tabular}{|c|c|c|c|c|c|c|}
\hline Parâmetros & & HG & & & HSP & \\
\hline $\begin{array}{c}\text { Tempos } \\
\text { (minutos) }\end{array}$ & $1^{\prime}$ & 5 & $10^{\prime}$ & $1^{\prime}$ & 5 & $10^{\prime}$ \\
\hline \multirow{3}{*}{$\begin{array}{c}\text { Dissoluçãa } \\
(\%)\end{array}$} & 85,03 & 85,07 & 85,16 & 85,12 & 85,11 & 84,98 \\
\hline & 85,03 & 85,06 & 85,16 & 85,11 & 85,12 & 84,97 \\
\hline & 85,03 & 85,07 & 85,16 & 85,06 & 85,05 & 84,92 \\
\hline $\begin{array}{c}\text { Média (\%) e } \\
\text { Desvio } \\
\text { Padrão }\end{array}$ & $\begin{array}{c}85,02 \pm \\
0,002\end{array}$ & $\begin{array}{c}85,06 \pm \\
0,008\end{array}$ & $\begin{array}{c}85,15 \pm \\
0,003\end{array}$ & $\begin{array}{c}85,08 \pm \\
0,030\end{array}$ & $\begin{array}{c}85,09 \pm \\
0,030\end{array}$ & $\begin{array}{c}84,95 \pm \\
0,002\end{array}$ \\
\hline
\end{tabular}

\subsection{DOSEAMENTO DO PARACETAMOL}

Para avaliação dos processos de mistura e perfil de dissolução fez-se necessário a elaboração da curva de calibração do paracetamol (Figuras 1 e 2). Através dos resultados das áreas do cromatograma foi obtida a equação da reta $y=31736 x+42787$. 


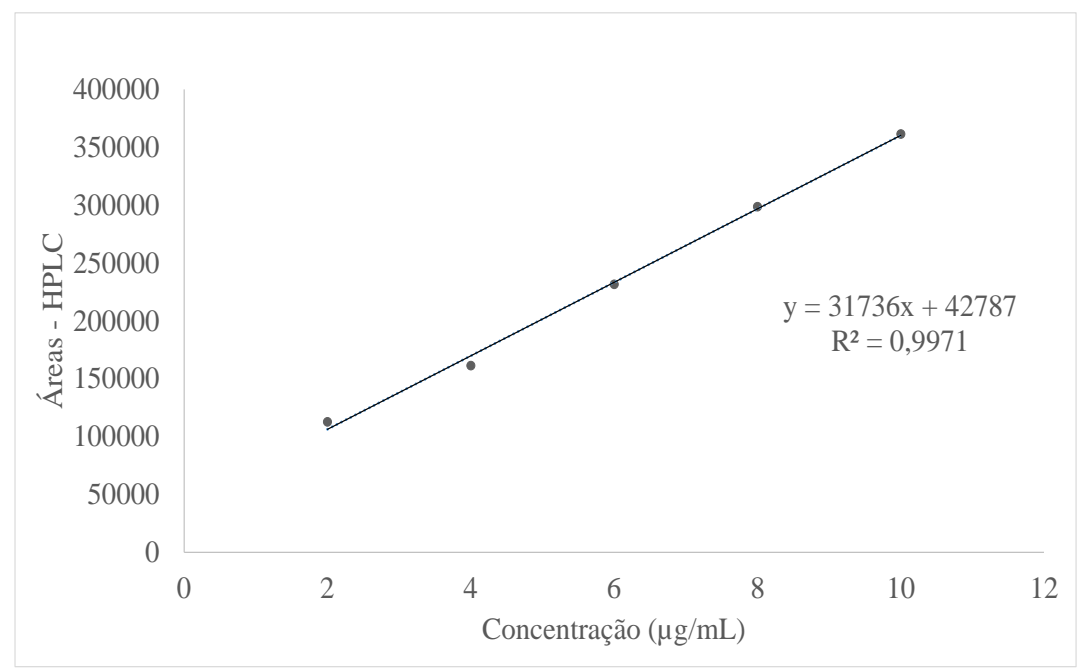

Figura 1: Curva padrão do paracetamol.

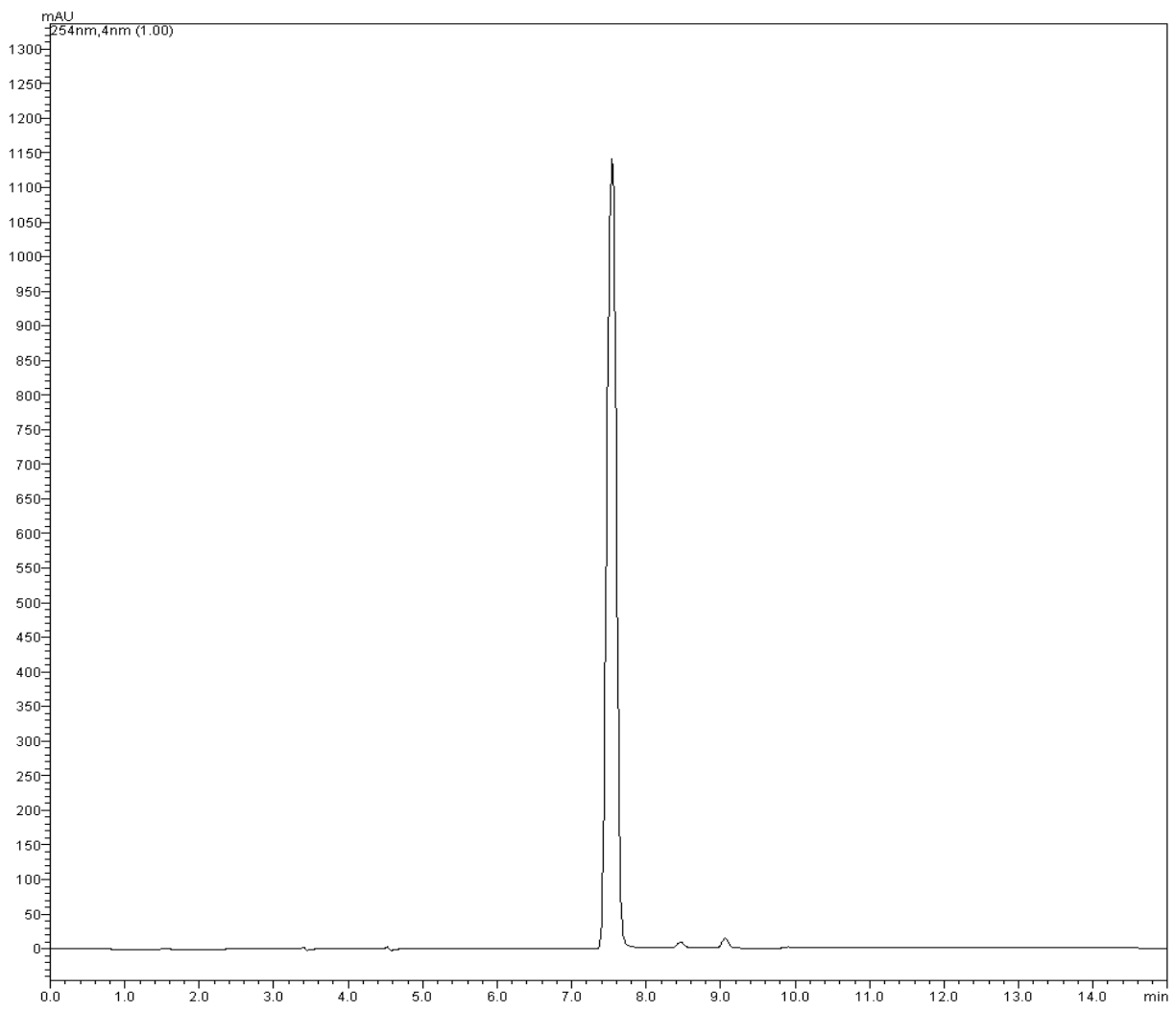

Figura 2: Cromatograma do paracetamol com o tempo de retenção de 7,5 minutos no comprimento de onda $254 \mathrm{~nm}$.

O teor de paracetamol nos diferentes métodos e tempos de homogeneização está descrito na Tabela 3. Os resultados obtidos para HG e HSP 1', 5' e 10' foram satisfatórios, tendo em vista que a Farmacopeia Brasileira $5^{\text {a }}$ edição estabelece como valor mínimo de princípio ativo $98 \%$ e máximo 102\% [13]. Diante disso, todos os métodos e tempos de homogeneização mostram-se aptos a serem utilizados como padrão pela farmácia magistral, visto que apresentaram teor de paracetamol dentro do limite preconizado. 
Tabela 3: Teor de paracetamol nos métodos de HG e HSP nos tempos de 1, 5 e 10 minutos.

\begin{tabular}{ccccccc}
\hline Parâmetros & \multicolumn{3}{c}{ HG } & \multicolumn{3}{c}{ HSP } \\
\hline Tempo (minutos) & 1 & $5^{\prime}$ & $10^{\prime}$ & 1 & 5 & $10^{\prime}$ \\
Teor médio (\%) & 101,3 & 101,2 & 101,5 & 101,6 & 101,4 & 101,6 \\
Desvio padrão & 0,40 & 0,09 & 0,23 & 0,04 & 0,04 & 0,04 \\
\hline
\end{tabular}

\section{CONCLUSÃO}

De acordo com os resultados obtidos no estudo, foi possível concluir baseado na Farmacopeia Brasileira $5^{\mathrm{a}}$ edição, que todos os métodos e tempos de homogeneização podem ser utilizados como padrão pela farmácia magistral na produção de cápsulas de paracetamol $(250 \mathrm{mg})$, otimizando o tempo de manipulação e garantindo a uniformidade das doses.

\section{REFERÊNCIAS BIBLIOGRÁFICAS}

1. Ferreira AO. Guia Prático da Farmácia Magistral. 3a. ed. São Paulo: Pharmabooks; 2008.

2. Oliveira AE, Hoffmann CL, Costa MP, Block LC. Análise e validação do procedimento de manipulação de cápsulas por encapsulamento manual por nivelamento. Revista de Ciências Farmacêuticas Básica e Aplicada. 2014;35(1):59-65.

3. Brasil. Ministério da Saúde. Agência Nacional de Vigilância Sanitária. RDC n ${ }^{\circ}$, de 08 de outubro de 2007. Dispõe sobre boas práticas de manipulação de preparações magistrais e oficinais para uso humano em farmácias. Diário Oficial da União, Brasília, 09 out. 2007. Seção 1.

4. Brasil. Ministério da Saúde. Agência Nacional de Vigilância Sanitária. RDC no 87, de 21 de novembro de 2008. Altera o Regulamento Técnico sobre Boas Práticas de Manipulação em Farmácias. Diário Oficial da União, Brasília, 21 nov. 2008.

5. Faria DP, Carvalho MM. Controle de qualidade de cápsulas de nifedipino manipuladas na região da zona da mata mineira. Revista Educação, Meio Ambiente e Saúde. 2012;5(1):82-93.

6. Brasil. Ministério da Saúde. Agência Nacional de Vigilância Sanitária. RDC no 33, de 19 de abril de 2000. Dispõe sobre o Regulamento Técnico sobre Boas Práticas de Manipulação de Medicamentos em farmácias e seus Anexos. Diário Oficial da União, Brasília, 19 abr. 2000.

7. Brasil. Ministério da Saúde. Agência Nacional de Vigilância Sanitária. RDC nº 214, de 12 de dezembro de 2006. Dispõe sobre Boas Práticas de Manipulação de Medicamentos para Uso Humano em farmácias. Diário Oficial da União, Brasília, 12 dez. 2006.

8. Allen Jr. LV, Popovich NG, Ansel HC. Formas farmacêuticas e sistemas de liberação de fármacos. $5^{\text {a }}$ ed. Porto Alegre: Artmed; 2007.

9. Ansel HC, Popovich NG, Ellen LV. Formas farmacêuticas e sistemas de liberação de fármacos. $8^{\mathrm{a}}$ ed. Porto Alegre: Artmed; 2007.

10. Wada MM, Ribeiro AF. Comparação entre métodos magistrais de homogeneização para preparação de cápsulas de alendronato de sódio: saco plástico x almofariz. Infarma-Ciências Farmacêuticas. 2014;26(1):27-34, doi:http://dx.doi.org/10.14450/2318-9312.v26.e1.a2014.pp27-34.

11. Ferreira AMN, Volpato NM, Freitas ZMF, Costa VA, Barros CM. Chiavegatto LFS, Quaresma CH. Validação do Processo de Mistura e Armazenamento de Pós para o Preparo de Cápsulas de Hidroxizine 25 mg. ANFARMAG Revista do Setor Farmacêutico Magistral. 2003;43:88-90.

12. Pinheiro GM, Benavide VG, Volpato NM, Cabral LM, Santos EP. Indicadores para controle de processo na manipulação de cápsulas em farmácia. Revista Brasileira de Farmácia. 2008;89(1):28-31.

13. Brasil. Agência Nacional de Vigilância Sanitária. Farmacopeia Brasileira - volume 2. $5^{\mathrm{a}}$ ed. Brasília (DF); 2010. 